\title{
Reactivity Studies of a Zirconium Methylidene Complex. Group Transfer and Methylenation Reactions
}

Takashi Kurogi, Masahiro Kamitani, Brian C. Manor, Patrick J. Carroll, and Daniel J. Mindiola*

Department of Chemistry, University of Pennsylvania, 231 South 34th Street, Philadelphia, Pennsylvania 19104

\section{Table of contents}

NMR spectroscopic data

Complex 2

S2-S3

Complex 3

S3-S4

Complex 4

S5-S7

Reaction of 1 with $\mathrm{CDCl}_{3}$

S7-S8

Reaction of 1 with cyclododecanone

S8-S9

Reaction of 1 with benzophenone

S9-S10

Tables of crystallographic data

Crystallographic data of $\mathbf{2}$

Crystallographic data of $\mathbf{3}$

S12

Crystallographic data of 4

$\mathrm{S} 13$ 


\section{NMR spectroscopic data}

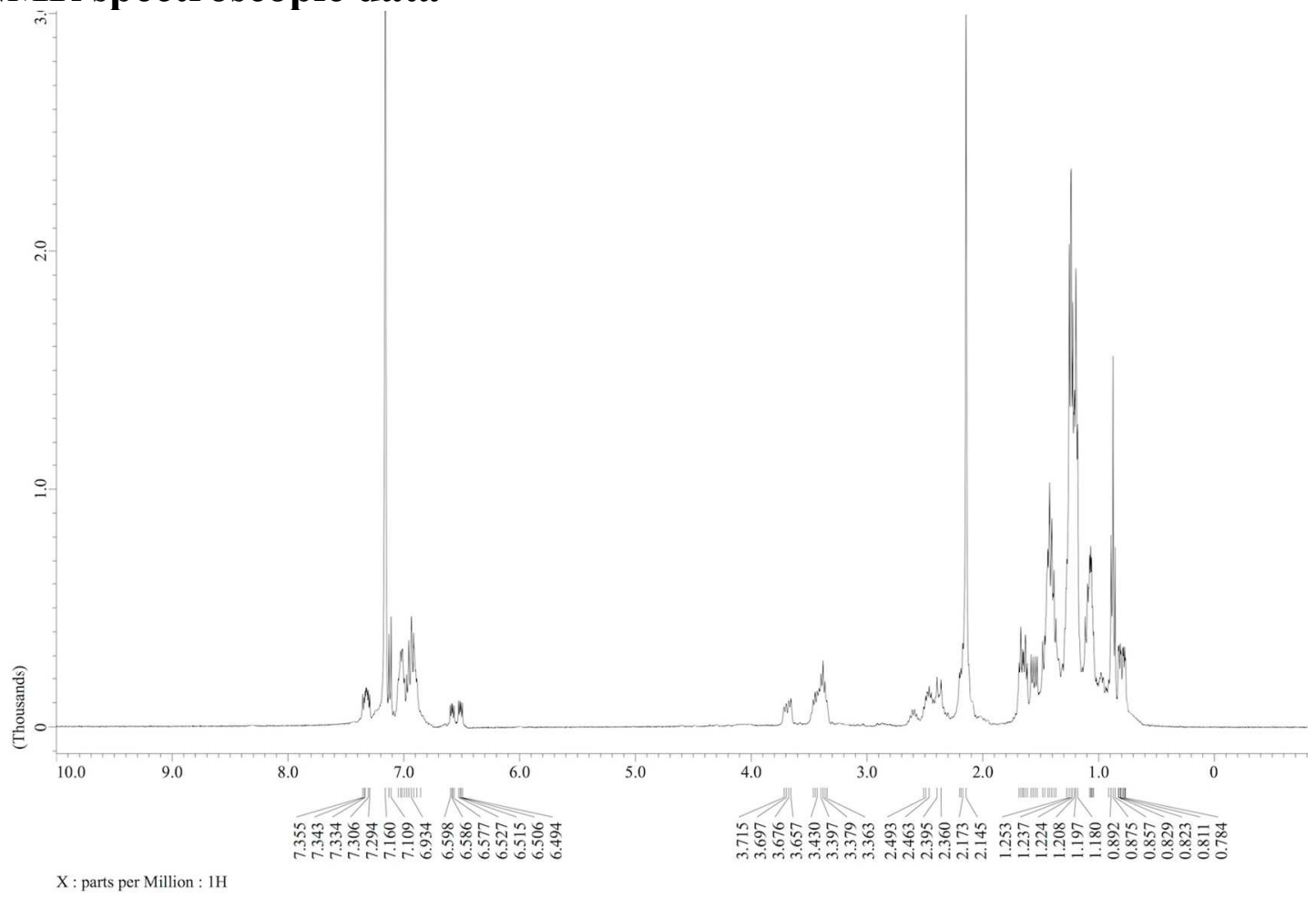

Figure S1. ${ }^{1} \mathrm{H}$ NMR spectrum of $2\left(400 \mathrm{MHz}\right.$, in Benzene- $d_{6}$, at $\left.300 \mathrm{~K}\right)$.

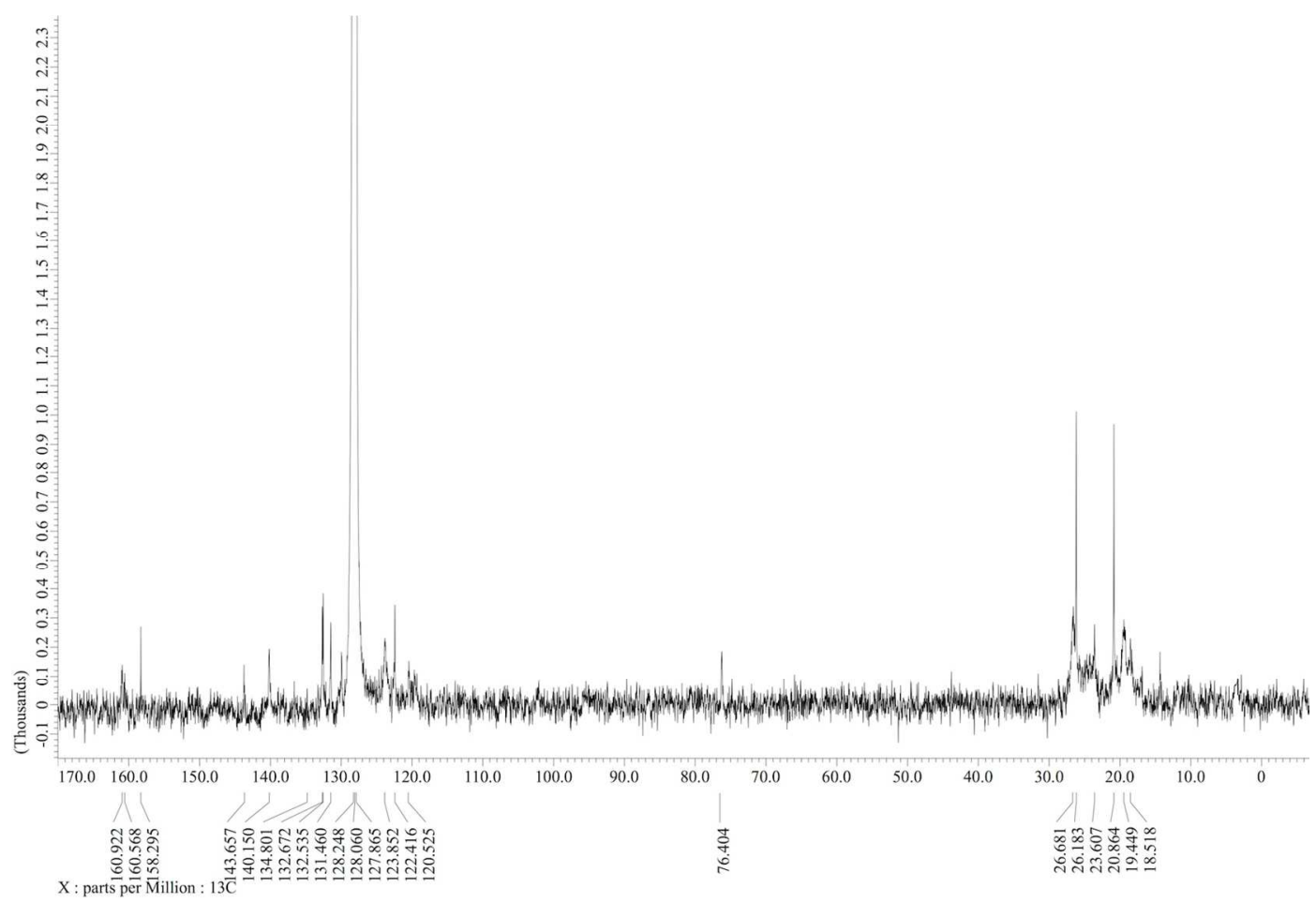

Figure S2. ${ }^{13} \mathrm{C}$ NMR spectrum of $2\left(126 \mathrm{MHz}\right.$, in Benzene- $d_{6}$, at $\left.300 \mathrm{~K}\right)$. 


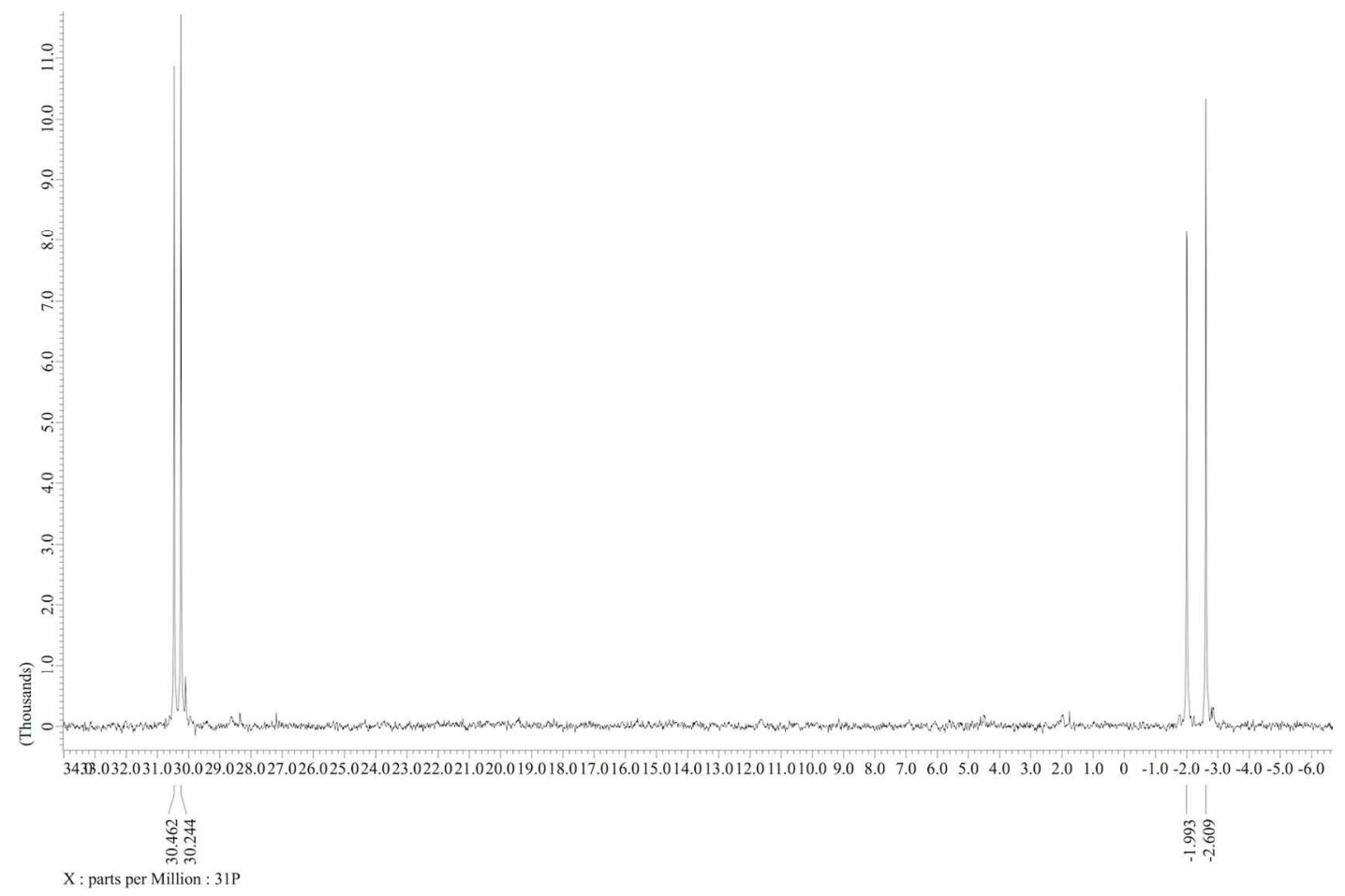

Figure S3. ${ }^{31} \mathrm{P}\left\{{ }^{1} \mathrm{H}\right\}$ NMR spectrum of $2\left(162 \mathrm{MHz}\right.$, in Benzene- $d_{6}$, at $\left.300 \mathrm{~K}\right)$.

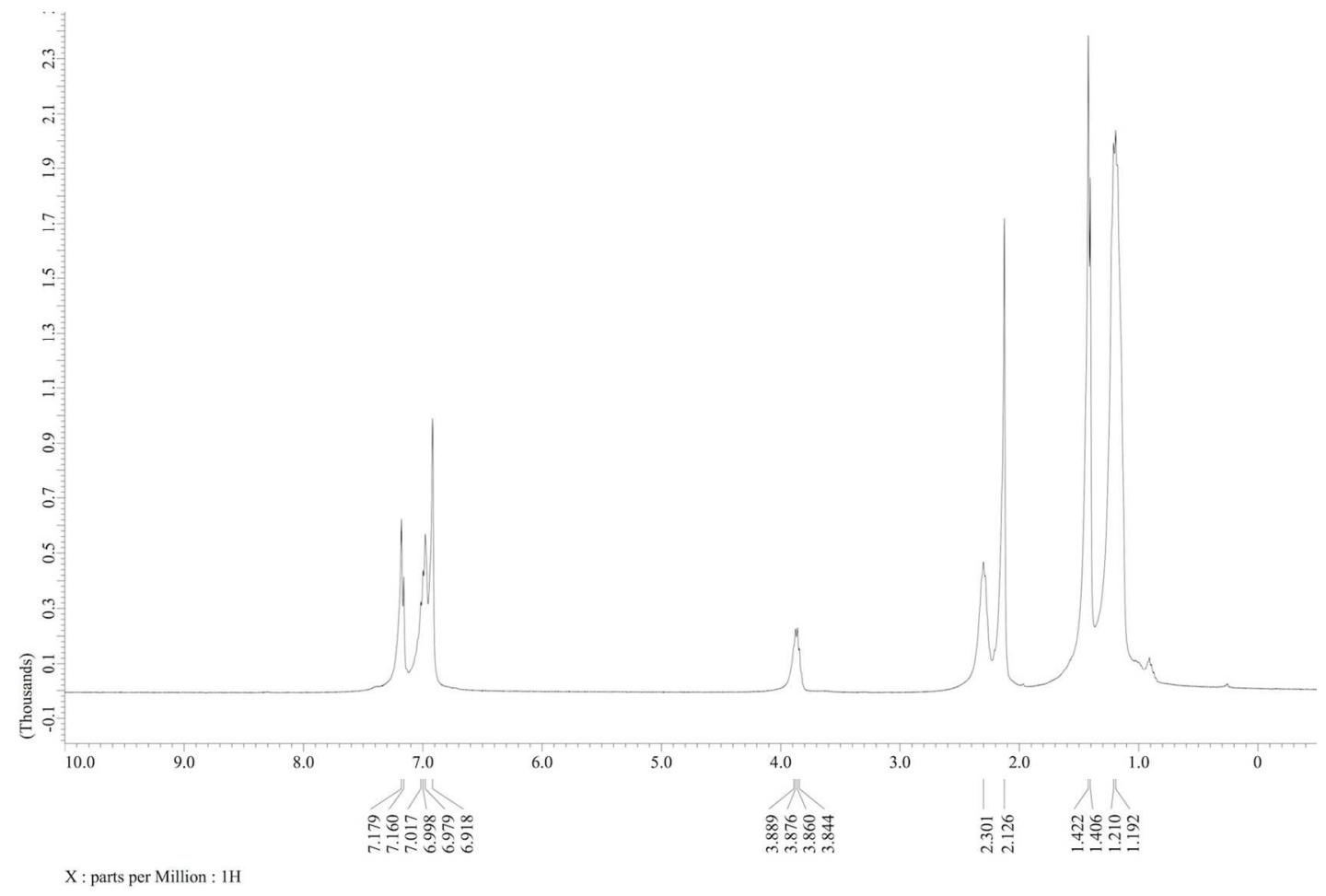

Figure S4. ${ }^{1} \mathrm{H}$ NMR spectrum of 3 (400 MHz, in Benzene- $d_{6}$, at $300 \mathrm{~K}$ ). 


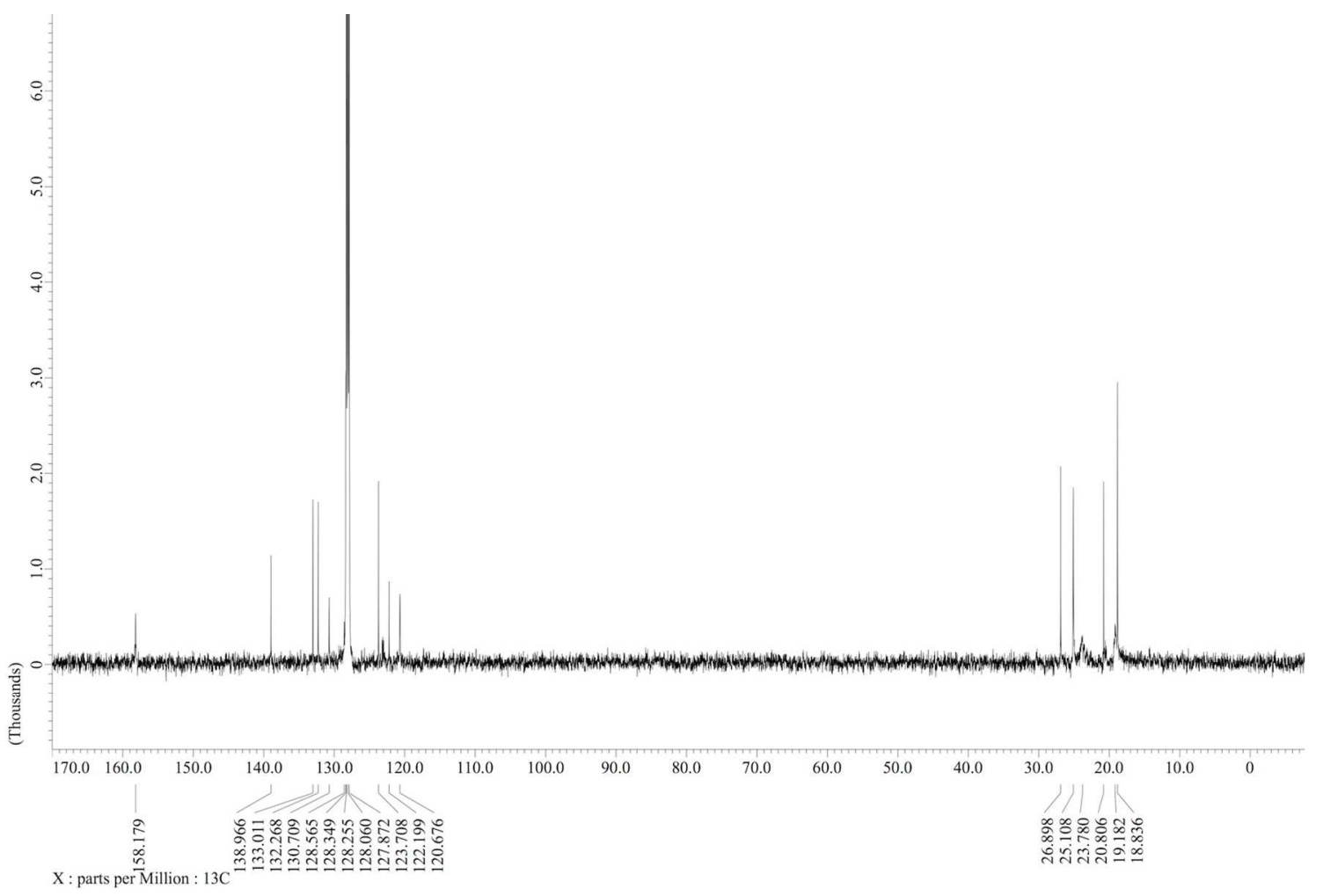

Figure S5. ${ }^{13} \mathrm{C}$ NMR spectrum of 3 (126 MHz, in Benzene- $d_{6}$, at $\left.300 \mathrm{~K}\right)$.

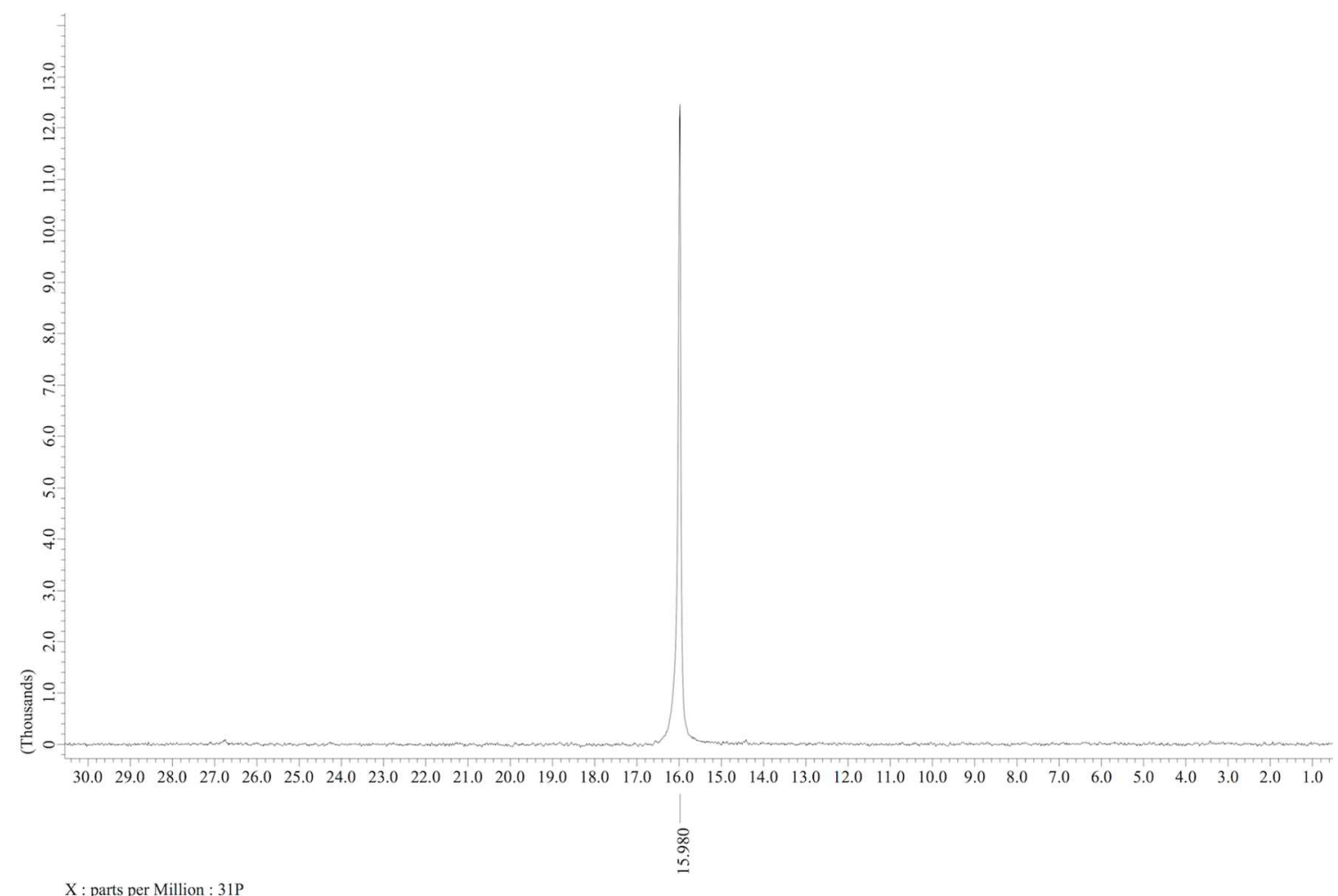

Figure S6. ${ }^{31} \mathrm{P}\left\{{ }^{1} \mathrm{H}\right\}$ NMR spectrum of $3\left(162 \mathrm{MHz}\right.$, in Benzene- $d_{6}$, at $\left.300 \mathrm{~K}\right)$. 


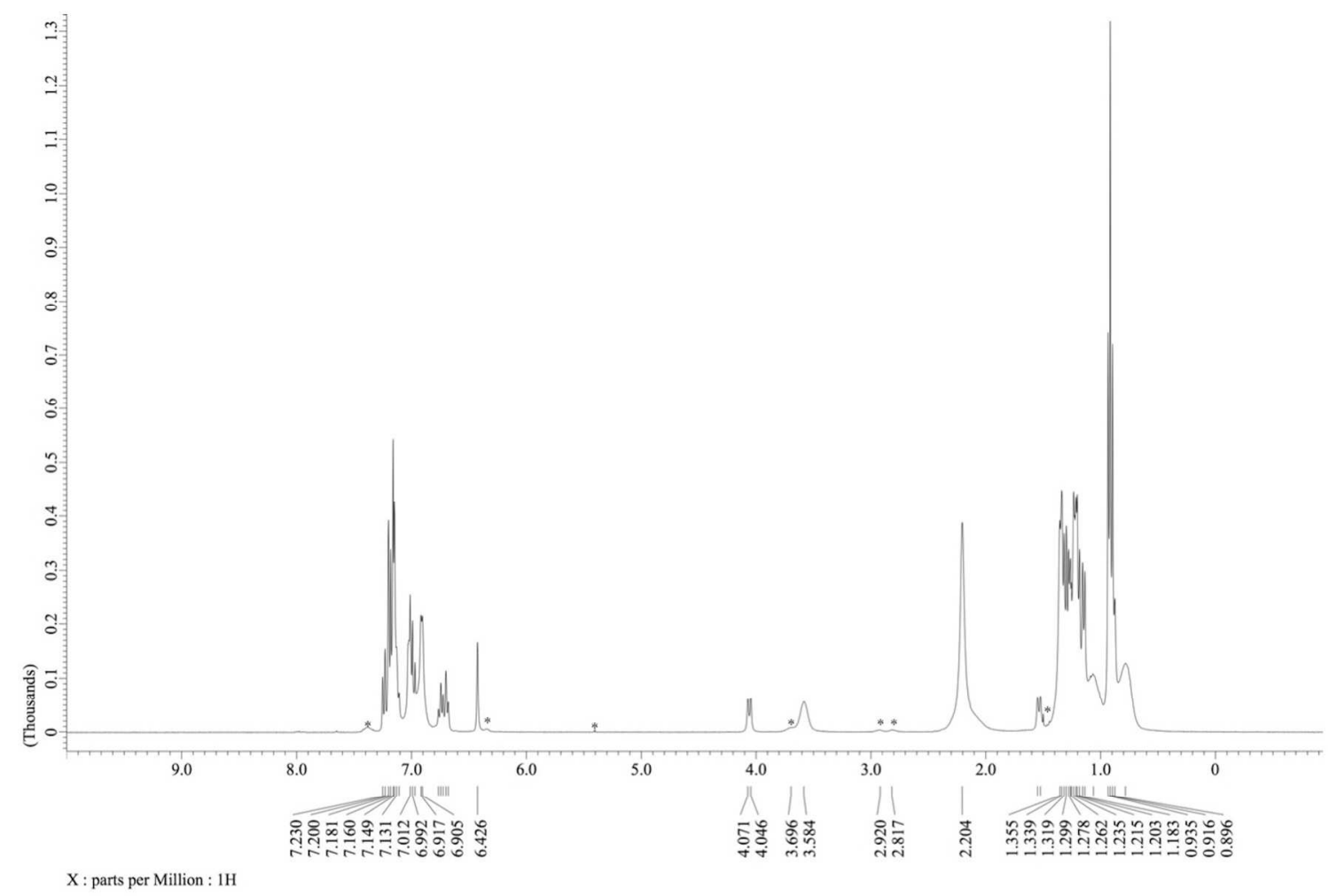

Figure S7. ${ }^{1} \mathrm{H}$ NMR spectrum of $4\left(360 \mathrm{MHz}\right.$, in Benzene- $d_{6}$, at $\left.300 \mathrm{~K}\right)$. *: Impurity.

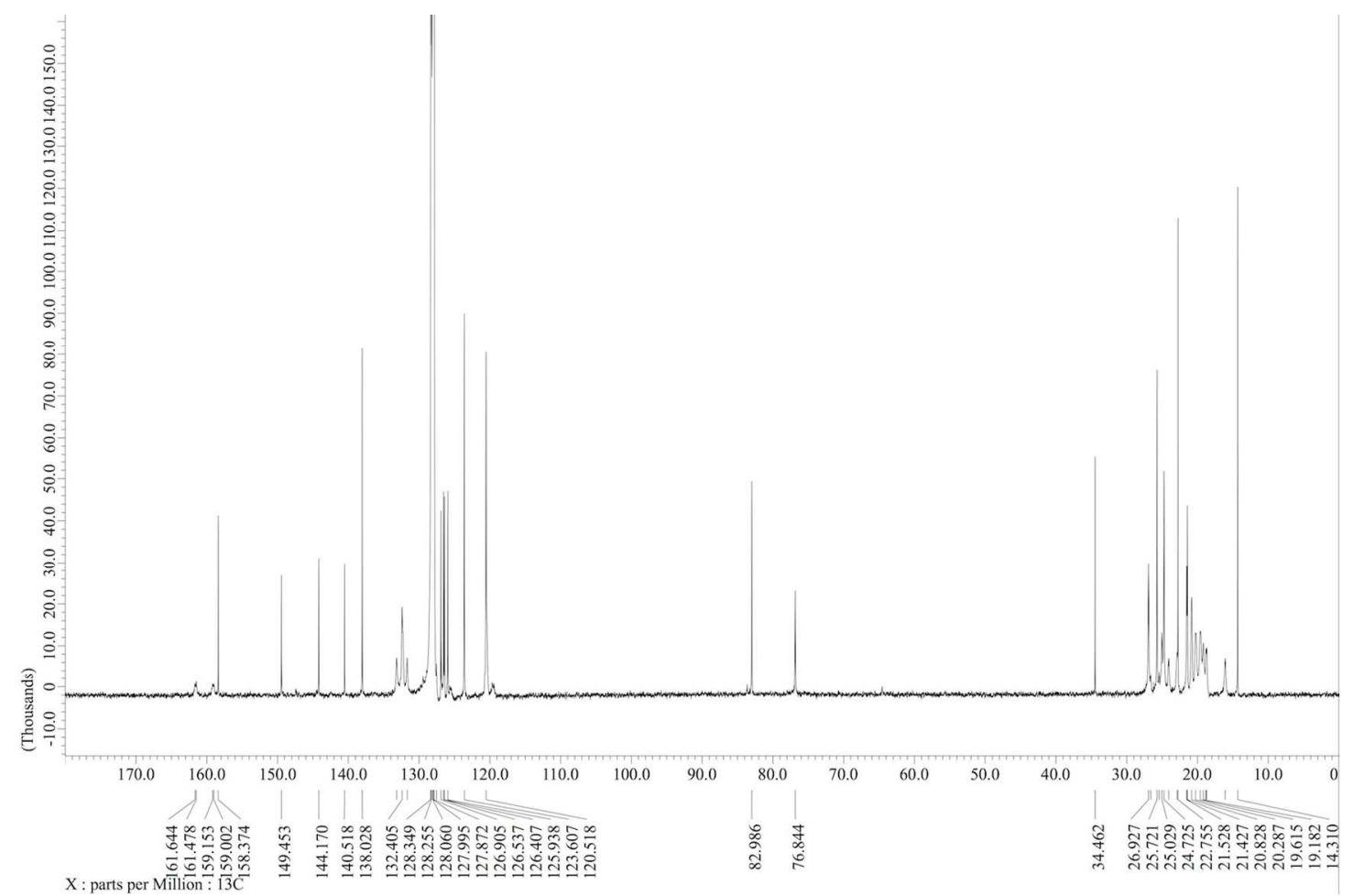

Figure S8. ${ }^{13} \mathrm{C}$ NMR spectrum of $4\left(126 \mathrm{MHz}\right.$, in Benzene- $d_{6}$, at $\left.300 \mathrm{~K}\right)$. *: Pentane. 


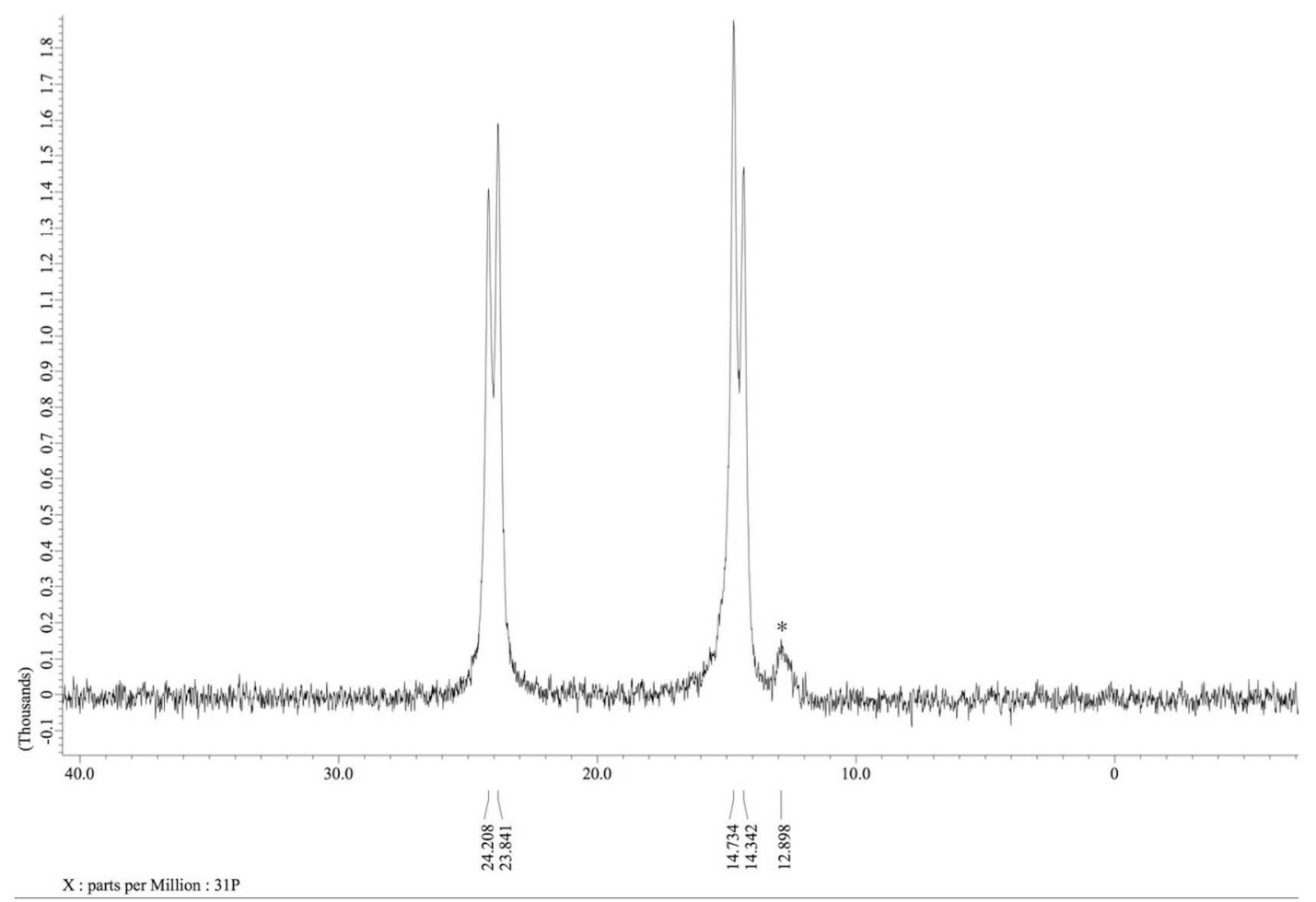

Figure S9. ${ }^{31} \mathrm{P}\left\{{ }^{1} \mathrm{H}\right\}$ NMR spectrum of $4\left(146 \mathrm{MHz}\right.$, in Benzene- $d_{6}$, at $\left.300 \mathrm{~K}\right)$. *: Impurity.

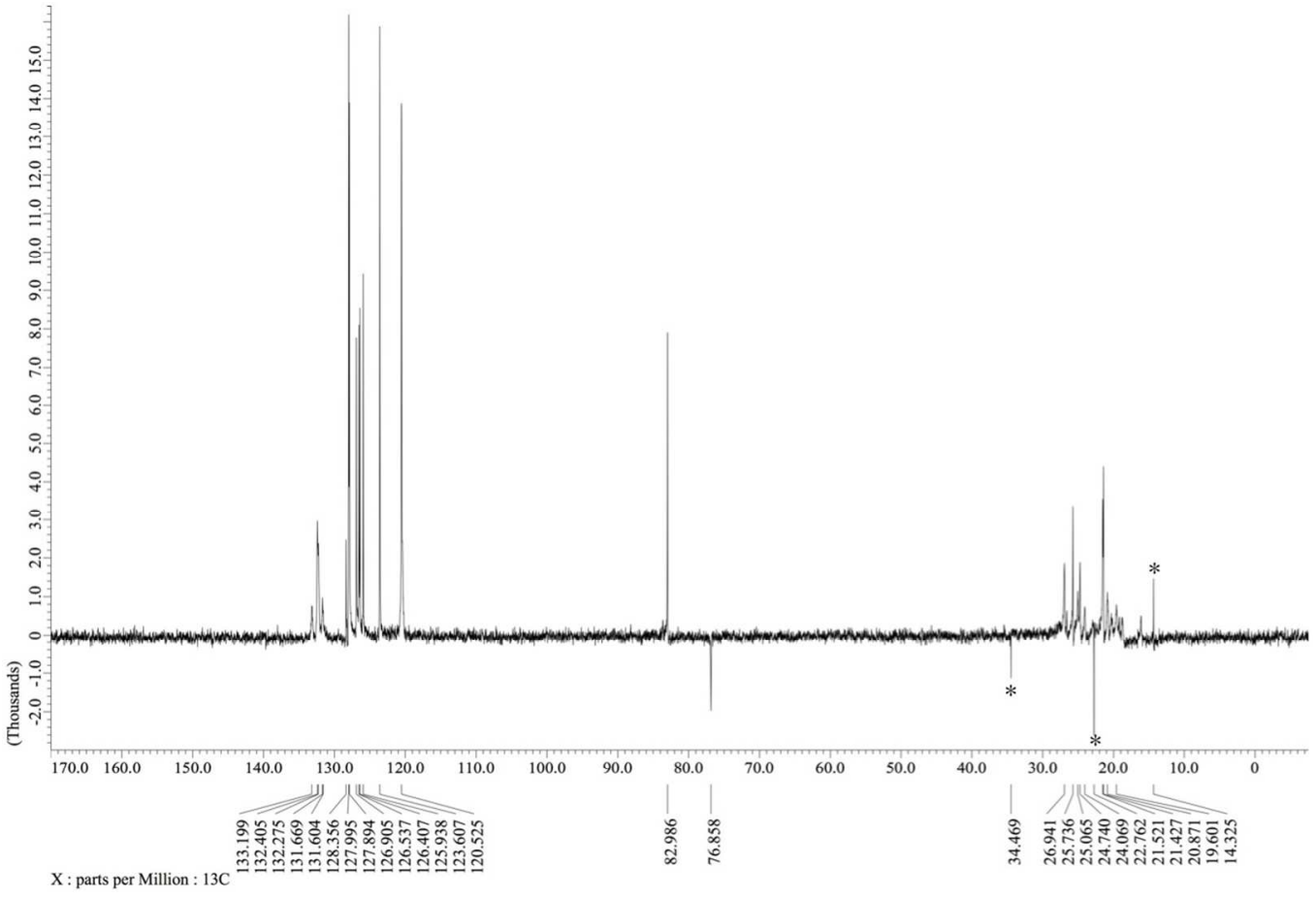

Figure S10. Dept $135{ }^{13} \mathrm{C}$ NMR spectrum of $4\left(126 \mathrm{MHz}\right.$, in Benzene- $d_{6}$, at $\left.300 \mathrm{~K}\right)$. *: Pentane. 


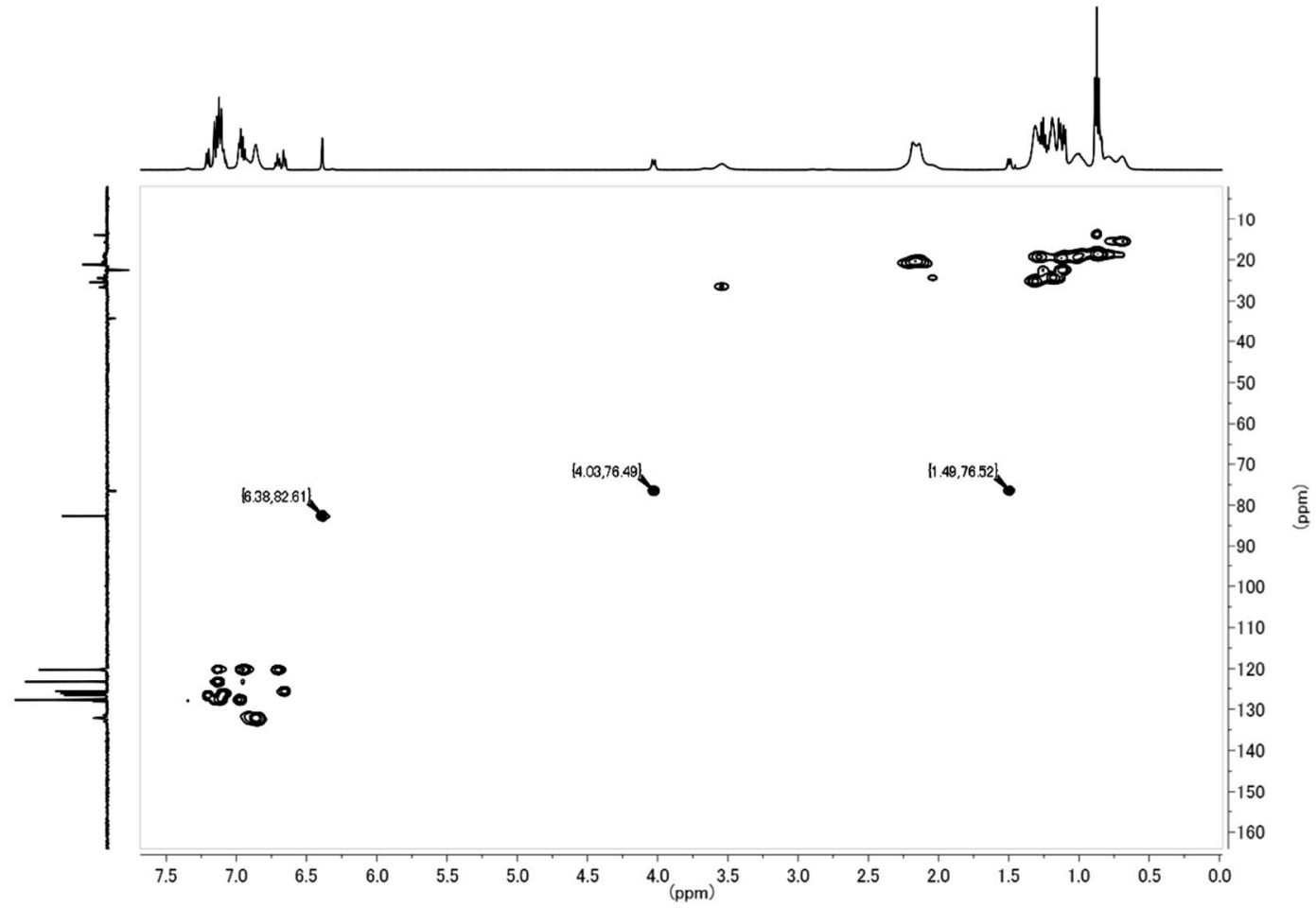

Figure S11. ${ }^{1} \mathrm{H}-{ }^{13} \mathrm{C}$ HSQC spectrum of $4\left(500 \mathrm{MHz}\right.$ in Benzene- $d_{6}$, at $300 \mathrm{~K}, \mathrm{X}$ axis: ${ }^{1} \mathrm{H}$, Y axis: ${ }^{13} \mathrm{C}$ NMR).

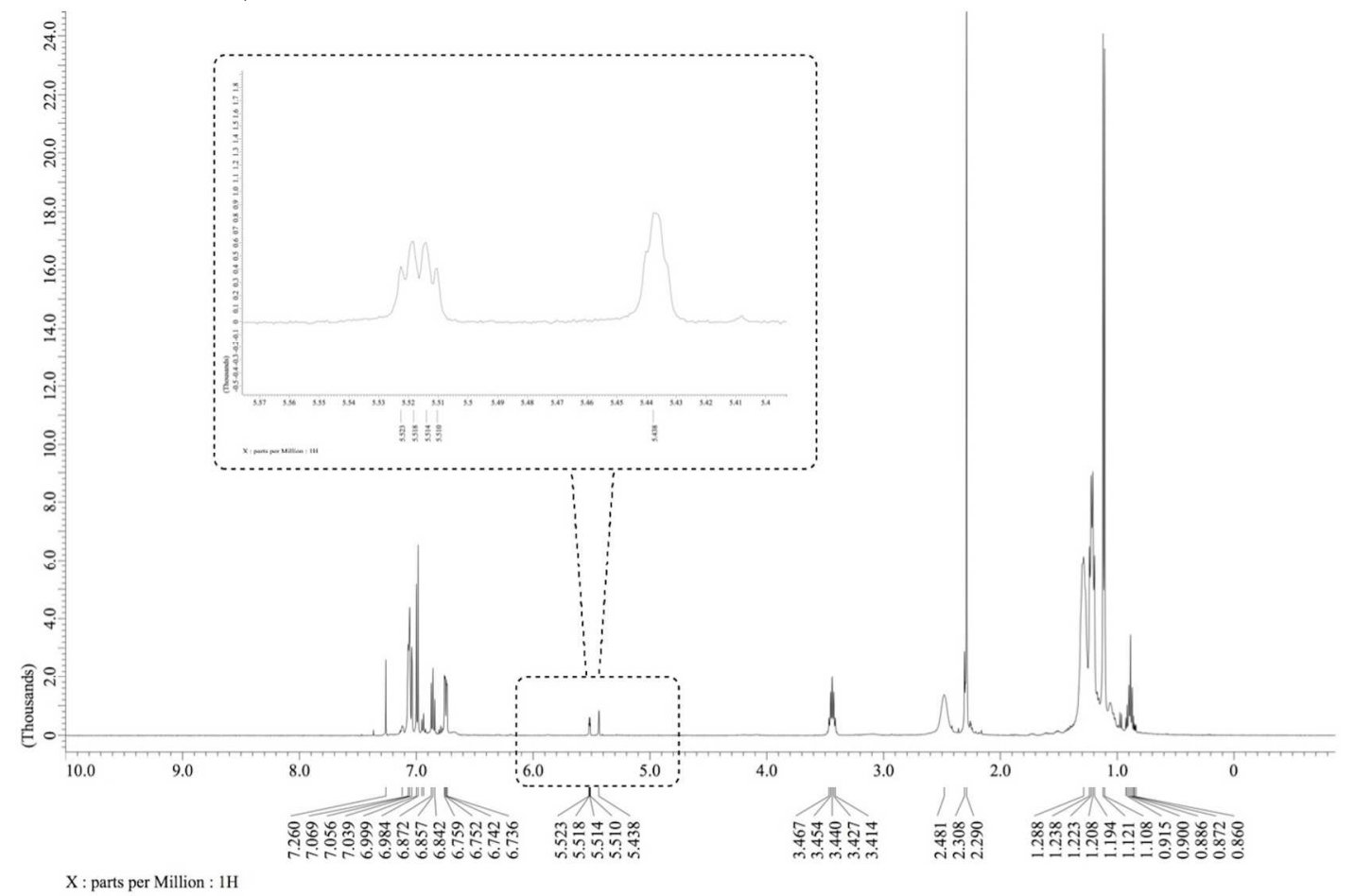

Figure S12. ${ }^{1} \mathrm{H}$ NMR spectrum of the reaction of 1 with $\mathrm{CDCl}_{3}(360 \mathrm{MHz}$, in Chloroform- $d_{1}$, at $300 \mathrm{~K}$ ). 


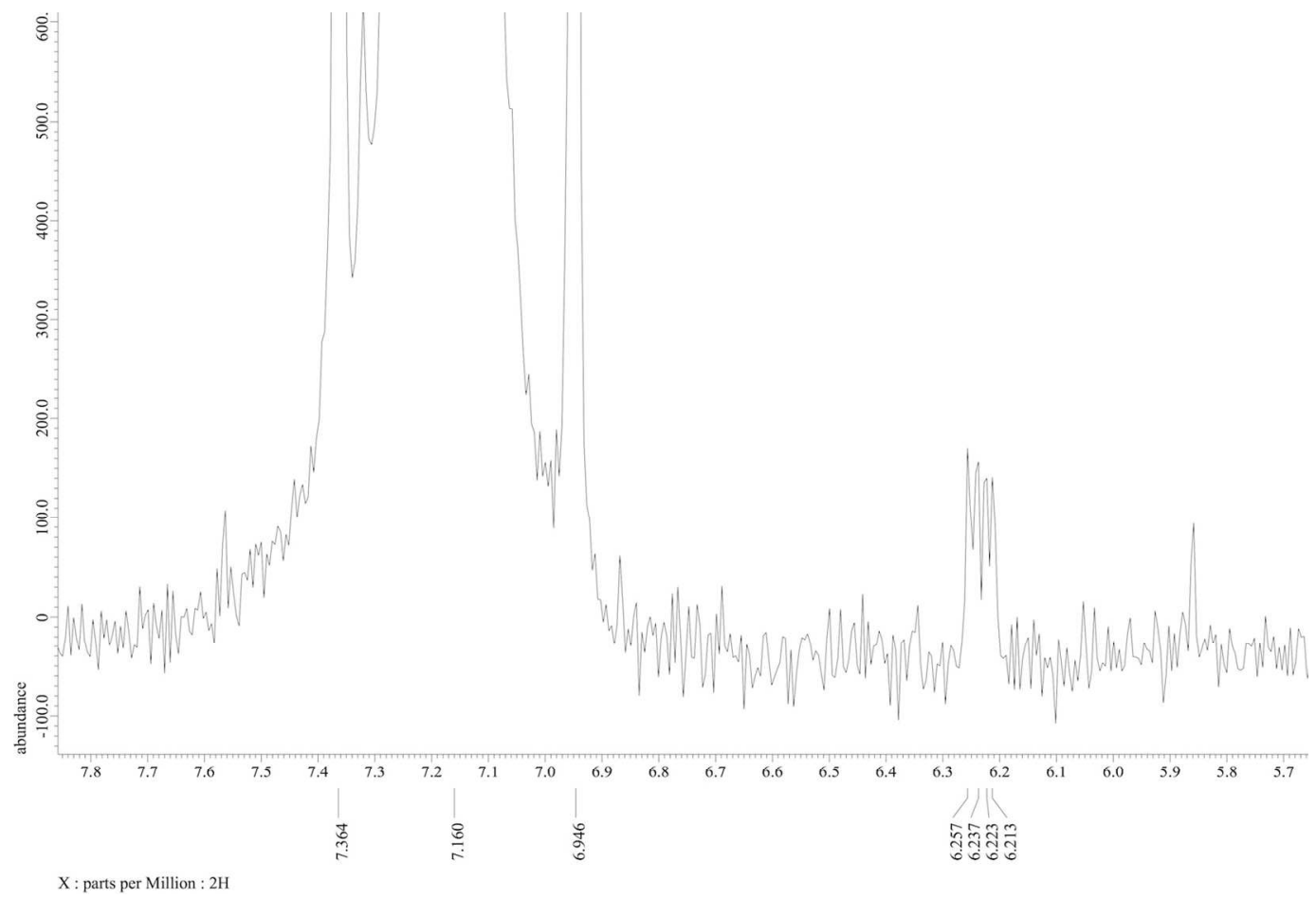

Figure S13. ${ }^{2} \mathrm{H}$ NMR spectrum of the reaction of 1 with $\mathrm{CDCl}_{3}(76.8 \mathrm{MHz}$, in Chloroform- $d_{1}$, at $\left.300 \mathrm{~K}\right)$.

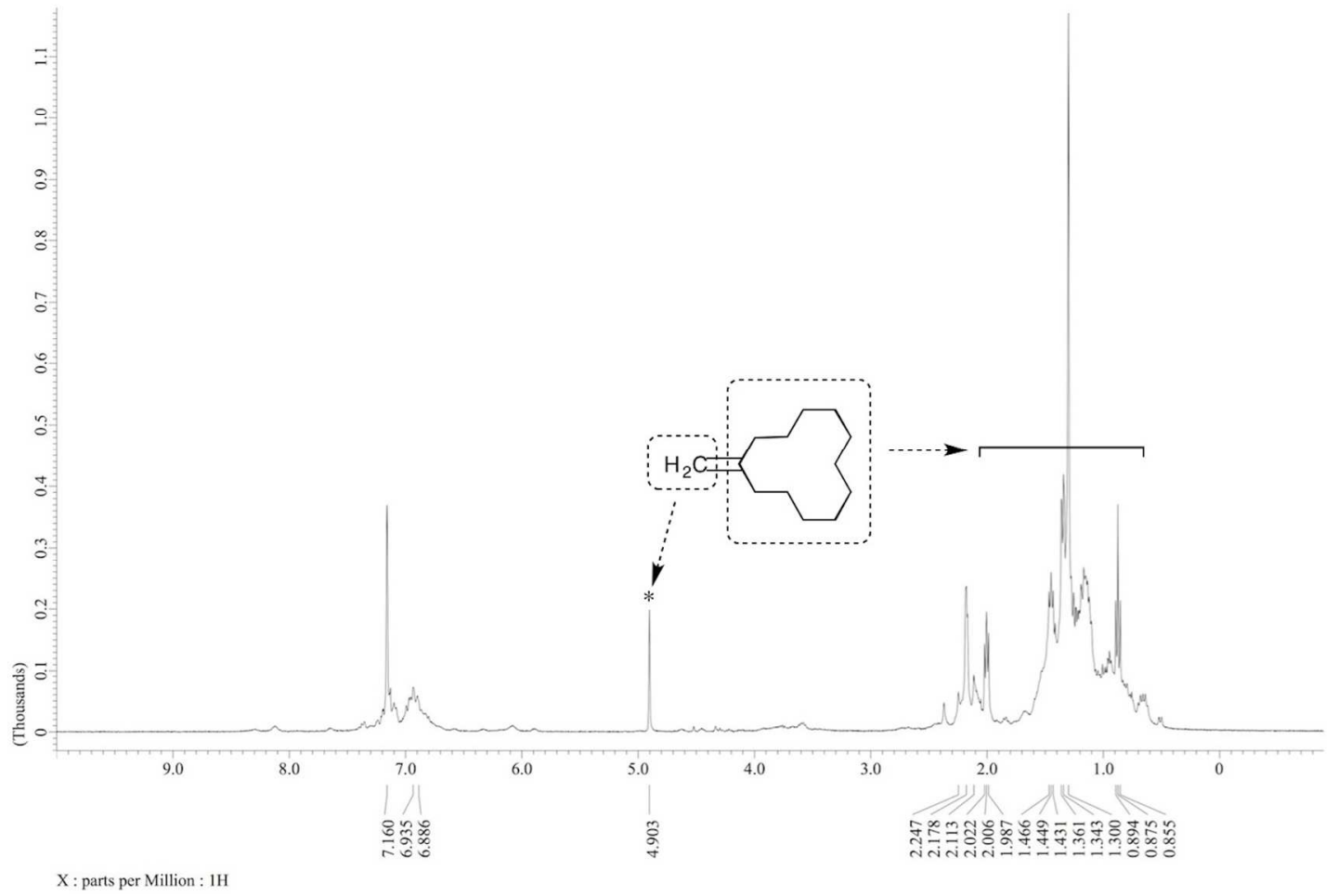

Figure S14. ${ }^{1} \mathrm{H}$ NMR spectrum of the reaction of 1 with cyclododecanone $(360 \mathrm{MHz}$, in Benzene- $d_{6}$, at $\left.300 \mathrm{~K}\right)$. 


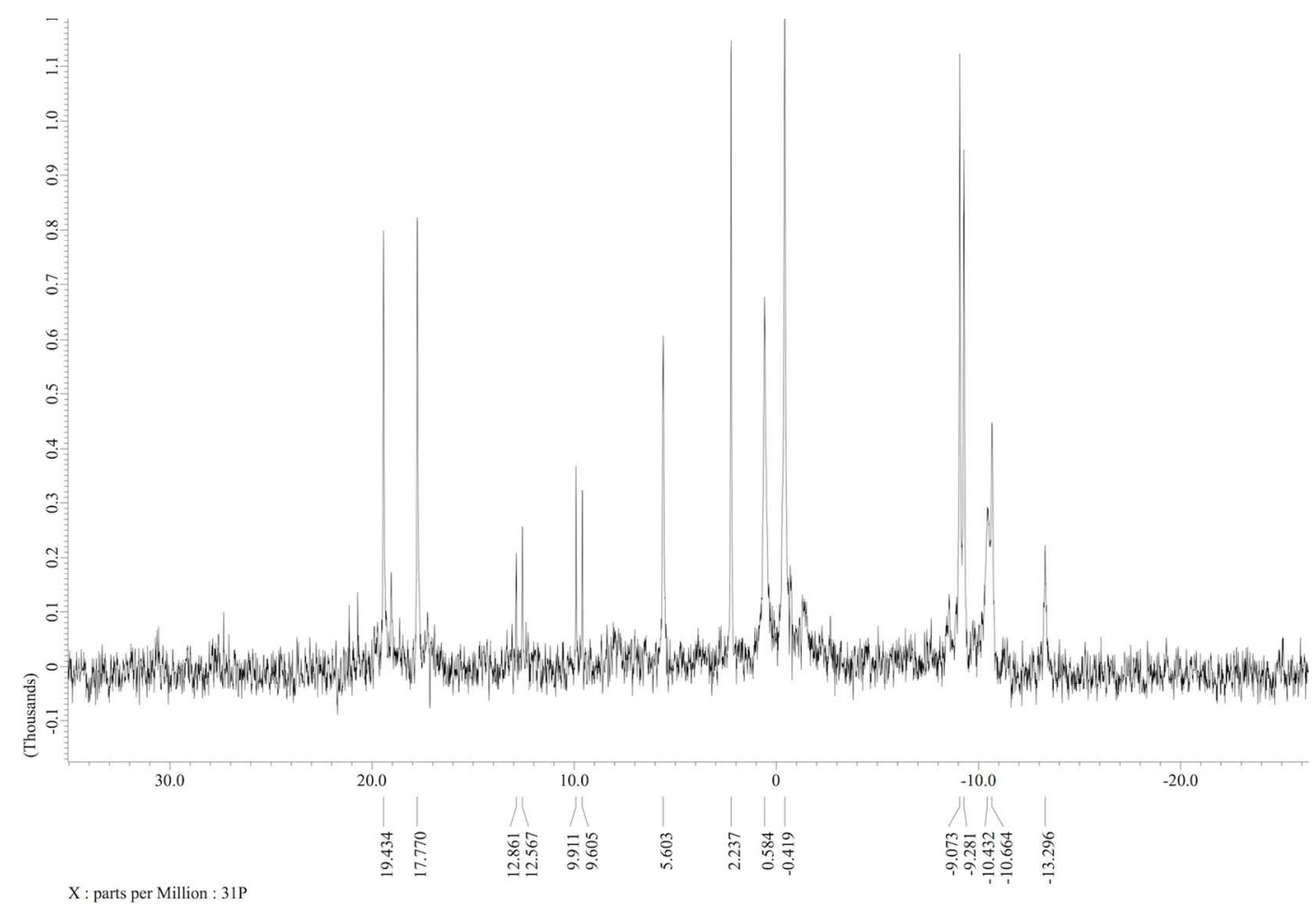

Figure S15. ${ }^{31} \mathrm{P}\left\{{ }^{1} \mathrm{H}\right\}$ NMR spectrum of the reaction of $\mathbf{1}$ with cyclododecanone (146 $\mathrm{MHz}$, in Benzene- $d_{6}$, at $\left.300 \mathrm{~K}\right)$.

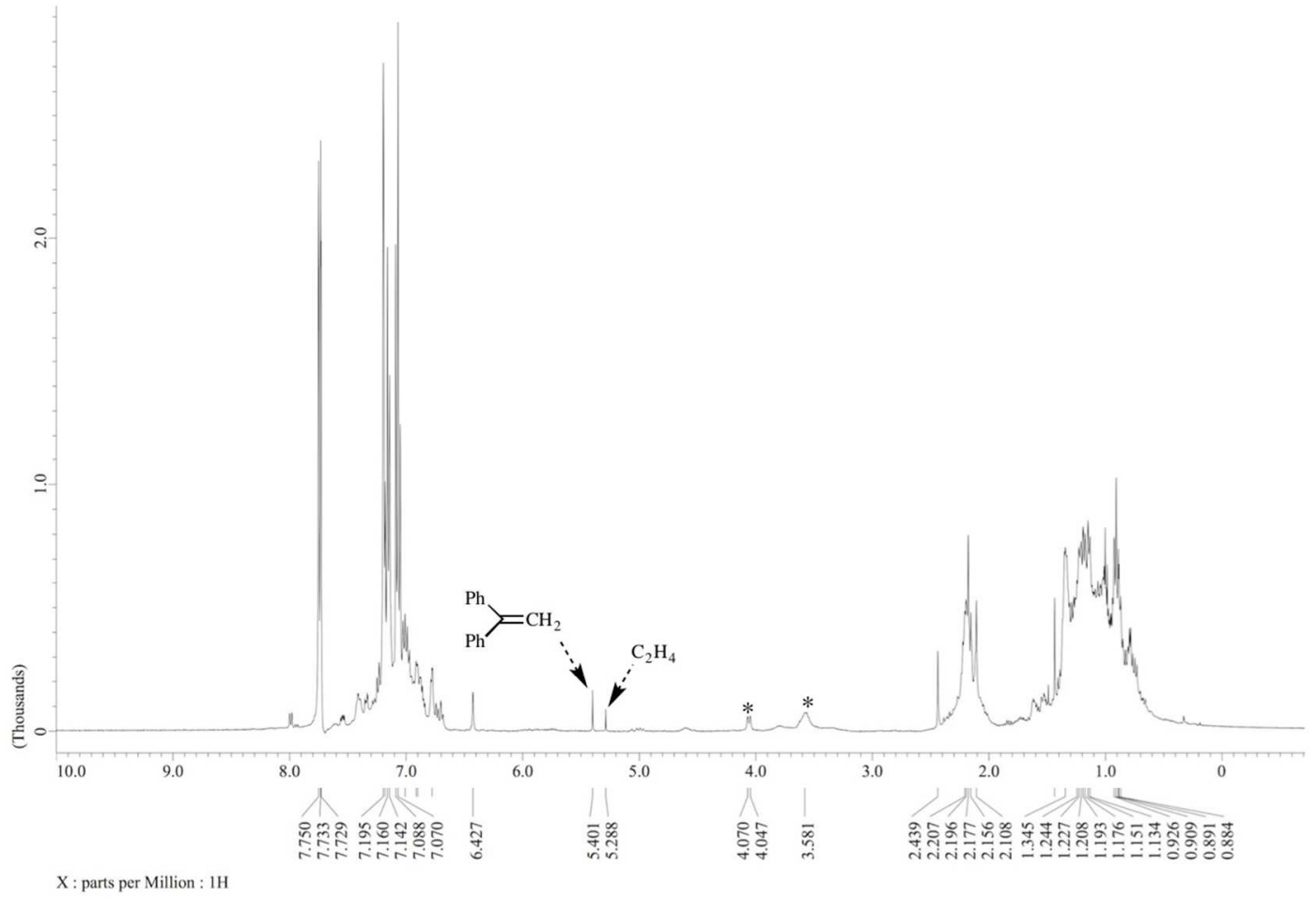

Figure S16. ${ }^{1} \mathrm{H}$ NMR spectrum of the reaction of 1 with benzophenone $(360 \mathrm{MHz}$, in Benzene- $d_{6}$, at $\left.300 \mathrm{~K}\right)$. *: Complex 4. 


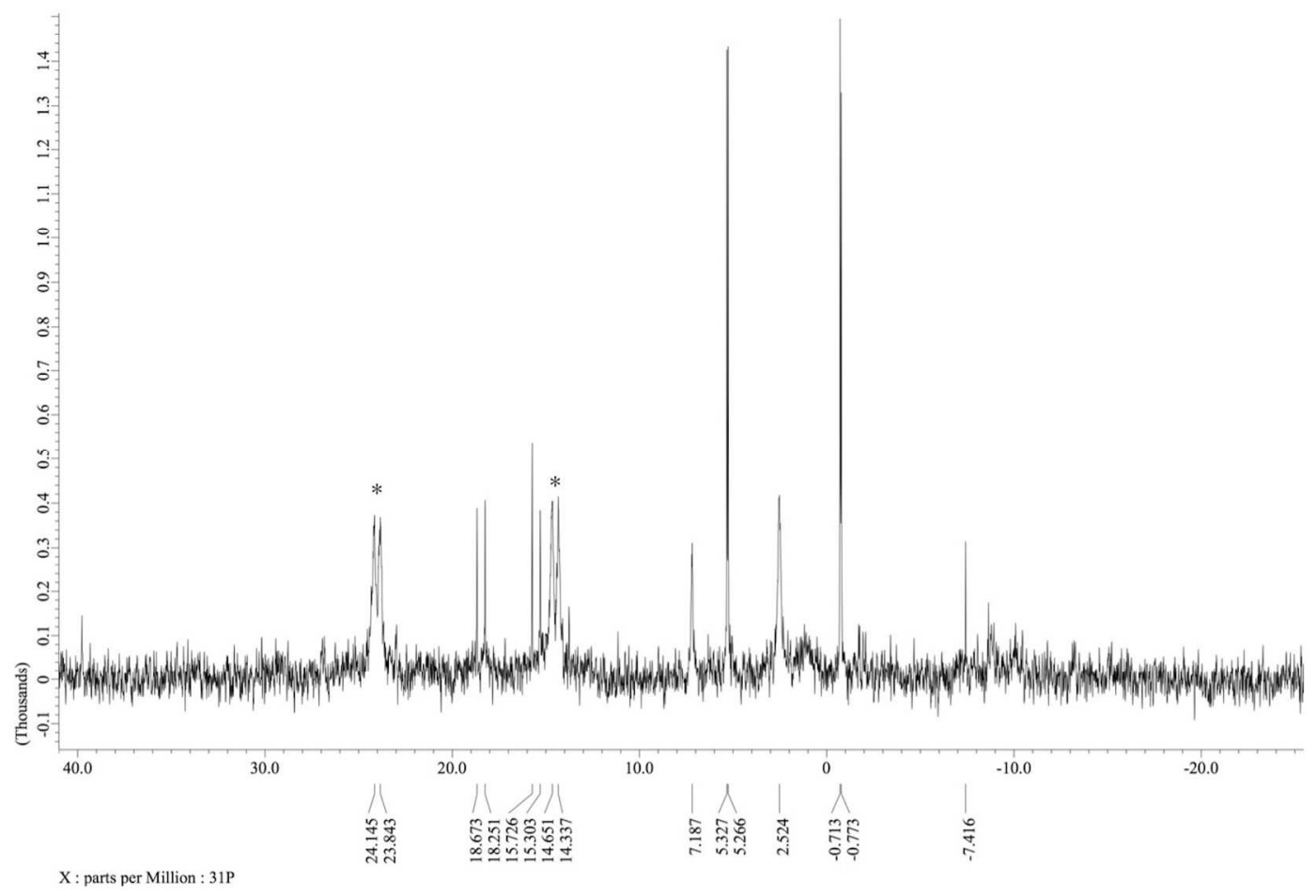

Figure S17. ${ }^{31} \mathrm{P}\left\{{ }^{1} \mathrm{H}\right\}$ NMR spectrum of the reaction of 1 with benzophenone $(162 \mathrm{MHz}$, in Benzene- $d_{6}$, at $\left.300 \mathrm{~K}\right) .{ }^{*}$ : Complex 4. 


\section{Tables of crystallographic data}

$\underline{\text { Table S1. Summary of Structure Determination of } 4 \cdot(\text { pentane) }} 3$

Empirical formula

Formula weight

Temperature

Wavelength

Crystal system

Space group

Cell constants:

$a$
$b$
$c$
$\alpha$
$\beta$
$\gamma$

Volume

Z

Density (calculated)

Absorption coefficient

$\mathrm{F}(000)$

Crystal size

Theta range for data collection

Index ranges

Reflections collected

Independent reflections

Completeness to theta $=27.55^{\circ}$

Absorption correction

Max. and min. transmission

Refinement method

Data / restraints / parameters

Goodness-of-fit on $F^{2}$

Final $\mathrm{R}$ indices $[\mathrm{I}>2 \operatorname{sigma}(\mathrm{I})]$

$\mathrm{R}$ indices (all data)

Largest diff. peak and hole
$\mathrm{C}_{95} \mathrm{H}_{154} \mathrm{~N}_{2} \mathrm{O}_{4} \mathrm{P}_{4} \mathrm{Zr}_{2}$

1694.51

100(1) K

$0.71073 \AA$

Triclinic

$P \mathrm{~T}$ (No. 2)

12.9074(5) $\AA$

12.9602(4) $\AA$

$15.8333(6) \AA$

$83.298(2)^{\circ}$

$86.298(2)^{\circ}$

$60.308(2)^{\circ}$

2285.08(15) $\AA^{3}$

1

$1.231 \mathrm{Mg} / \mathrm{m}^{3}$

$0.347 \mathrm{~mm}^{-1}$

910

$0.38 \times 0.32 \times 0.03 \mathrm{~mm}^{3}$

1.295 to $27.497^{\circ}$

$-16 \leq \mathrm{h} \leq 16,-16 \leq \mathrm{k} \leq 16,-20 \leq 1 \leq 20$

66679

$10462[R($ int $)=0.0213]$

$99.7 \%$

Semi-empirical from equivalents

0.7456 and 0.7140

Full-matrix least-squares on $F^{2}$

10462 / 0 / 536

1.085

$R_{1}=0.0357, w R_{2}=0.0955$

$R_{1}=0.0399, w R_{2}=0.0986$

0.812 and -0.713 e. $\AA^{-3}$ 


\section{Table S2. Summary of Structure Determination of 3}

Empirical formula

Formula weight

Temperature

Wavelength

Crystal system

Space group

Cell constants:

$a$
$b$
$c$
$\alpha$
$\beta$
$\gamma$

Volume

Z

Density (calculated)

Absorption coefficient

$\mathrm{F}(000)$

Crystal size

Theta range for data collection

Index ranges

Reflections collected

Independent reflections

Completeness to theta $=27.55^{\circ}$

Absorption correction

Max. and min. transmission

Refinement method

Data / restraints / parameters

Goodness-of-fit on $F^{2}$

Final $\mathrm{R}$ indices $[\mathrm{I}>2 \operatorname{sigma}(\mathrm{I})]$

$\mathrm{R}$ indices (all data)

Largest diff. peak and hole

Flack Parameter
$\mathrm{C}_{38} \mathrm{H}_{57} \mathrm{NOCl}_{2} \mathrm{P}_{2} \mathrm{Zr}$

767.90

100(1) K

$0.71073 \AA$

Monoclinic

$P 2_{1}$ (No. 4)

$12.071(3) \AA$

17.963(4) $\AA$

$17.976(5) \AA$

$90^{\circ}$

$90.045(11)^{\circ}$

$90^{\circ}$

3897.7(16) $\AA^{3}$

4

$1.309 \mathrm{Mg} / \mathrm{m}^{3}$

$0.531 \mathrm{~mm}^{-1}$

1616

$0.24 \times 0.09 \times 0.07 \mathrm{~mm}^{3}$

3.044 to $27.497^{\circ}$

$-15 \leq \mathrm{h} \leq 15,-23 \leq \mathrm{k} \leq 23,-23 \leq 1 \leq 23$

106855

$17900[R($ int $)=0.0657]$

$99.8 \%$

Semi-empirical from equivalents

0.7456 and 0.7063

Full-matrix least-squares on $F^{2}$

17900 / 67 / 887

1.061

$R_{1}=0.0272, w R_{2}=0.0522$

$R_{1}=0.0301, w R_{2}=0.0532$

0.279 and -0.318 e. $\AA^{-3}$

$-0.037(10)$ 
Table S3. Summary of Structure Determination of $4 \cdot$ (pentane)

Empirical formula

Formula weight

Temperature

Wavelength

Crystal system

Space group

Cell constants: $a$

$b$

$c$

$\alpha$

$\beta$

$\gamma$

Volume

Z

Density (calculated)

Absorption coefficient

$\mathrm{F}(000)$

Crystal size

Theta range for data collection

Index ranges

Reflections collected

Independent reflections

Completeness to theta $=27.55^{\circ}$

Absorption correction

Max. and min. transmission

Refinement method

Data / restraints / parameters

Goodness-of-fit on $F^{2}$

Final $\mathrm{R}$ indices $[\mathrm{I}>2 \operatorname{sigma}(\mathrm{I})]$

$\mathrm{R}$ indices (all data)

Largest diff. peak and hole

Flack Parameter
$\mathrm{C}_{57} \mathrm{H}_{81} \mathrm{NO}_{2} \mathrm{P}_{2} \mathrm{Zr}$

965.38

100(1) K

$0.71073 \AA$

Orthorhombic

Pca2 $_{1}$ (No. 29)

$31.3061(15) \AA$
$10.3460(5) \AA$
$16.5525(9) \AA$
$90^{\circ}$
$90^{\circ}$
$90^{\circ}$

$5361.2(5) \AA^{3}$

4

$1.196 \mathrm{Mg} / \mathrm{m}^{3}$

$0.304 \mathrm{~mm}^{-1}$

2064

$0.15 \times 0.10 \times 0.10 \mathrm{~mm}^{3}$

1.301 to $27.498^{\circ}$

$-40 \leq \mathrm{h} \leq 40,-13 \leq \mathrm{k} \leq 11,-21 \leq 1 \leq 21$

61558

$12168[R($ int $)=0.1057]$

$99.6 \%$

Semi-empirical from equivalents

0.7456 and 0.6062

Full-matrix least-squares on $F^{2}$

12168 / 63 / 610

1.025

$R_{1}=0.0552, w R_{2}=0.1180$

$R_{1}=0.0890, w R_{2}=0.1343$

1.564 and -0.446 e. $\AA^{-3}$

$-0.04(2)$ 